\title{
Value of Virtual Institutes $\&$ the Synergy of Science and Exploration
}

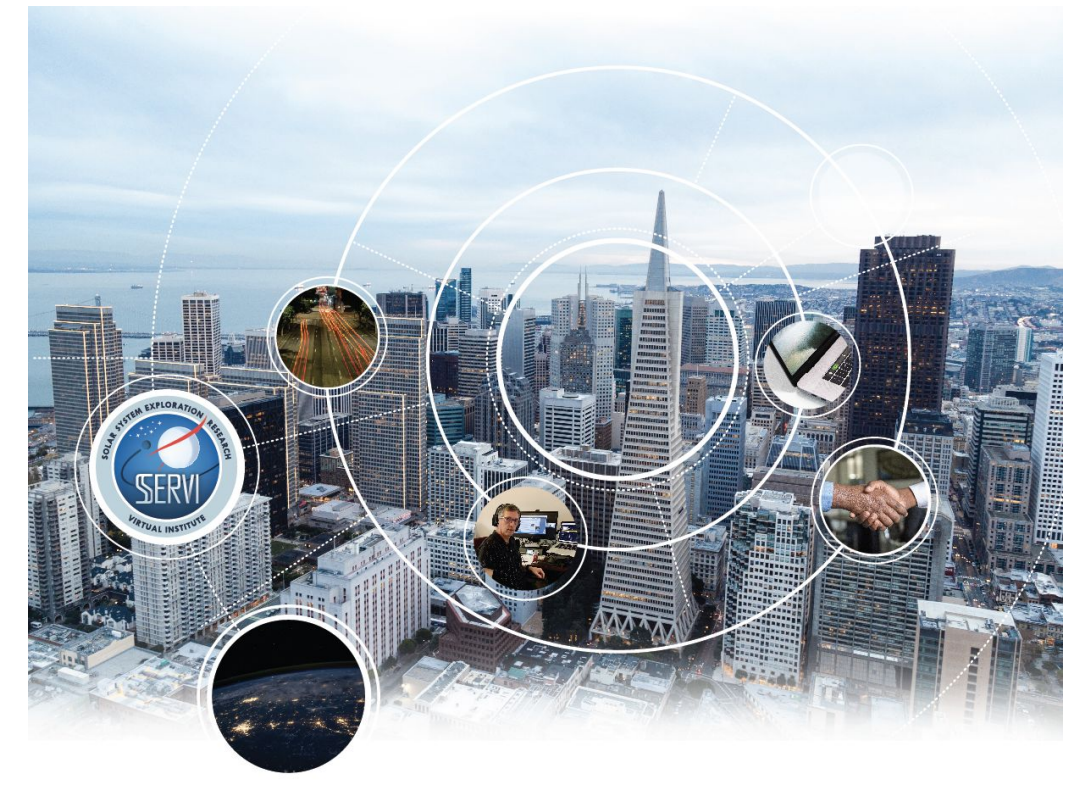

\section{Authors:}

Greg Schmidt, SSERVI Director, (650) 604-2611, gregory.schmidt@nasa.gov

Kristina Gibbs, SSERVI Deputy Director, (650) 604-0654, kristina.gibbs@nasa.gov

\section{Endorsers:}

Carle Pieters, Brown University

Ben Bussey, Johns Hopkins University Applied Physics Lab

Esther Beltran, University of Central Florida

Daniel Britt, University of Central Florida

Jack Burns, University of Colorado, Boulder

Adrienne Dove, University of Central Florida

Amy Fagan, Western Carolina University, Chair, Lunar Exploration Analysis Group

Jeffrey Gillis-Davis, Washington University, St. Louis

Timothy Glotch, Stonybrook University, New York

Jennifer Heldmann, NASA Ames Research Center

Amanda Hendrix, Planetary Science Institute

Mihaly Horanyi, University of Colorado, Boulder

Rosemary Killen, NASA Goddard Space Flight Center

Rachel Klima, Johns Hopkins University Applied Physics Lab

David Kring, Lunar \& Planetary Institute

Tobin Munsat, University of Colorado, Boulder

Clive R. Neal, University of Notre Dame, IN

Thom Orlando, Georgia Institute of Technology

Alex Parker, Southwest Research Institute

Andy Rivkin, Johns Hopkins University Applied Physics Lab

Nicholas Schmerr, University of Maryland, College Park

Faith Vilas, Planetary Science Institute 


\section{Introduction}

This White Paper describes virtual institutes as an essential aspect of the planetary science portfolio, using the Solar System Exploration Research Virtual Institute (SSERVI) as an example. Virtual institutes offer key attributes that advance space science and exploration in unique ways. In focusing peer-reviewed research teams on key agency problems, virtual institutes employ relatively large, multidisciplinary efforts that can tackle more complex problems that last longer than typical R\&A grants. Research collaborations are encouraged between a diverse set of funded teams and international partners, resulting in many unanticipated cross-team efforts. Virtual institutes are an excellent mechanism to bridge disciplines within the Science Mission Directorate (SMD), the Human Exploration and Operations Mission Directorate (HEOMD) and Science and Technology Mission Directorate (STMD). SSERVI's charter occupies a unique niche at the intersection of SMD and HEOMD and is an excellent example of bridging the two directorates. SSERVI's past and future contributions to Artemis and other human exploration programs can help integrate science and exploration objectives. SSERVI is also closing key knowledge gaps that will help establish a permanent, sustained human presence in space and allow humans and robots to safely conduct lunar and interplanetary missions.

\section{SSERVI Introduction}

SSERVI is a virtual institute at the intersection of science and exploration. SSERVI [sservi.nasa.gov] began as the NASA Lunar Science Institute (NLSI) in 2008 and was broadened in 2013 to include human exploration targets beyond the Moon. SSERVI is comprised of a global network of dispersed, interdisciplinary researchers collaborating to address key fundamental science and human exploration questions. The scope of SSERVI research includes NASA's top near-term destinations for human explorers, including NASA's bold plan to go forward to the Moon and beyond through the Artemis Program.

Founded on the principle that science enables exploration, and exploration enables science, SSERVI research areas span from fundamental science to human exploration [Fig 1]. The institute is focused primarily on exploration science, the science leading to, and benefiting from, human exploration beyond our home planet. The SSERVI community pursues a variety of fundamental questions ranging from basic science to applied research. SSERVI's research thus goes beyond pure science with the flexibility to assist HEOMD on questions directly applicable to the Artemis program, including how we can use the Moon as a stepping stone to prepare for missions to Mars.

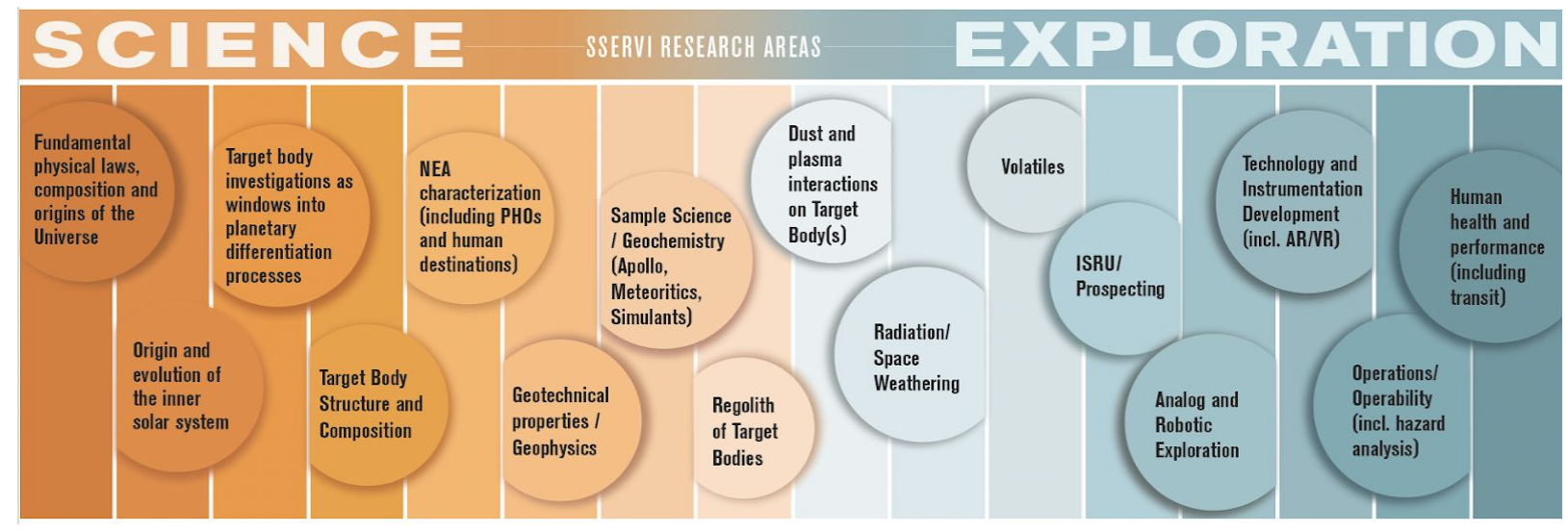




\section{Figure 1: Range of Current SSERVI Research Areas}

SSERVI research is jointly funded by NASA's SMD and HEOMD to address complex, multi-faceted science questions and key human spaceflight concerns. NASA funds competitively selected U.S. teams, while the SSERVI Central office at NASA Ames Research Center in Silicon Valley exercises overall leadership and management of the institute. SSERVI Central provides direction and guidance to its teams and international, and commercial partners, supports the planetary science and exploration communities and public engagement, and connects all with videoconferencing and other collaborative tools. SSERVI is also deeply committed to using the virtual institute model to take steps to enable positive change in equity, diversity, and inclusion and established the SSERVI Equity, Diversity, and Inclusion (EDI) Focus Group [https://sservi.nasa.gov/equity-diversity-and-inclusion-focus-group/] with the goal of identifying and undertaking specific actions to manifest immediate positive change within the space science and exploration communities.

Presently, approximately 260 researchers are funded at various levels through the SSERVI teams, with an additional 120 domestic and 50 international collaborators. Students are a major component of each SSERVI funded team as well. As of January 1st, 2020, SSERVI teams published over 900 peer-reviewed publications, with over $16 \%$ inter-team collaborations, $20 \%$ having student authorship, and 14\% with international collaboration. The NLSI publication list and a searchable SSERVI Publication Database are available on the SSERVI website [https://sservi.nasa.gov/science-library/].

Organizationally, SSERVI consists of 4 key elements: (1) SSERVI Central: Institute leadership and management based at NASA Ames Research Center; (2) Research Teams: Currently 13 research teams at institutions across the United States; each team has a Principal Investigator and is itself comprised of a number of different institutions; (3) International Partners: 11 international partners, each involved in key studies in exploration science with collaborative activities with SSERVI; (4) Solar System Treks Portal: An online visualization tool managed through SSERVI and developed at JPL incorporating many layers of observational and other data; Moon Trek is being used to help determine landing sites for Artemis and by VIPER for mission planning. The Trek Suite allows astronomers, citizen scientists, and students to study a wide range of features on several planetary bodies.

In addition to facilitating key research elements, programs such as SSERVI can multiply their impact for NASA through supporting the greater research community, training the next generation, and engaging the public in NASA's mission. As examples of support that a virtual institute can provide to the research community, SSERVI hosts focus groups and the NASA Exploration Science Forum [https://sservi.nasa.gov/nesf/]. The focus groups address topical areas of community interest and have yielded a multitude of white papers, as well as specific studies and reports that have helped inform NASA on key strategic objectives and in establishing new directions [https://sservi.nasa.gov/focus-groups/]. The NESF is hosted yearly by SSERVI and is open to the planetary science, human exploration, astrophysics, and heliophysics communities with a focus on SSERVI's targeted bodies; the Moon, near-Earth asteroids and the moons of Mars. The NESF is a direct link between NASA and its programs, and the scientists and engineers working to enable a sustained human presence in space. This meeting is typically produced as an in-person event with virtual options for those who cannot attend in person, and with archived recordings made available on-demand on the NESF website.

A foundational element of the virtual institute model has been training the next generation in a host of research-centered activities. SSERVI solicitations encourage and fund 
postdoctoral and student participation as a core part of the greater research program resulting in numerous graduate students and postdoctoral fellows in SSERVI teams. SSERVI supports team development of student programs for all levels in the lab and field to provide authentic research that support pathways to science careers, and sponsors the LunGradCon [http://impact.colorado.edu/lungradcon/], a conference specifically for the next generation of lunar scientists that is organized by graduate students and held in conjunction with the NESF. As an institute, SSERVI also focuses funding on NASA Post-doctoral Fellows to bridge the work of 2-3 SSERVI teams and thus accelerate collaborative inter-team activities. And where possible, SSERVI actively invites NextGen scientists to participate in leadership roles in meetings and conferences and supports their travel awards for NESF and LEAG.

A virtual institute is also an excellent tool for NASA to inspire the next generation and inform the public of NASA's plans and accomplishments. The SSERVI Central office and the SSERVI teams are funded to enable the science activation/citizen science/public engagement ecosystem that NASA has created, again bridging NASA programs. As an example, SSERVI has used a diverse portfolio of activities to inspire and inform the public through scientific, educational, and public lectures and events; schools and under-served communities; training teachers; developing braille books [https://sservi.nasa.gov/tactile/]; supporting robotic competitions, and working with citizen scientists to provide input to various programs such as the Fireballs in the Sky program [http://fireballsinthesky.com.au/].

Yearly, NLSI and now SSERVI, publishes an Annual Report that captures the extensive research results, collaborations, community, and public engagement from the SSERVI teams, the efforts of SSERVI Central as well as collaborations with international partners. The reports going back to 2010 are available at https://sservi.nasa.gov/science-library/.

\section{Structure and Value of Virtual Institutes}

Bringing together different scientific disciplines and orchestrating them to focus on common complicated problems (e.g., the Moon) has tremendous value for NASA.

Exploration science requires a variety of expertise to resolve issues and develop operational mission concepts. The virtual institute model focuses on individual research team efforts and facilitates collaboration on critical questions, resulting in a multifaceted, problem-solving, approach. Integrating industry and academic partnerships with NASA research and development has resulted in an exchange of insights between the human exploration and space science communities and helped pave a clearer path to resolving issues that are key for future human exploration. In support of these endeavors, SSERVI has advanced transformative lunar science, developed mission, instrument, and technology concepts specifically related to exploration science, and has contributed to mission planning and operations.

The structure of virtual institutes provides long-term, stable research environments. Each SSERVI team is funded for 5 years, which supports research continuity and allows for flexibility to pursue new scientific questions as discoveries are made. This principle was established by the late Nobel Prize winner Dr. Barry Blumberg, who served as the founding director of the NASA Astrobiology Institute. He famously told the first research teams, "Ultimately, I expect you not to do what's in your proposal," alluding to the fact that long-term efforts often end much differently than they started, and in the process advance and even change their respective fields.

Each cohort of funded teams is staggered approximately every 2.5 years, which allows overlap between established teams and new teams (Fig. 2). This overlapping nature of the teams 
provides a continuity to the institute, fosters collaboration, and helps ensure an alignment in core research areas, such that investigations are strategically focused to avoid duplicative efforts between teams. Sharing students, facilities and resources also reduces cost and enhances institute effectiveness. With stable funding it also provides secure, long-term funding for graduate students and postdoctoral positions. As compared to small, individually awarded grants, this kind of interdisciplinary, multi-institutional research would be difficult or would not have been possible in the absence of this funding model.

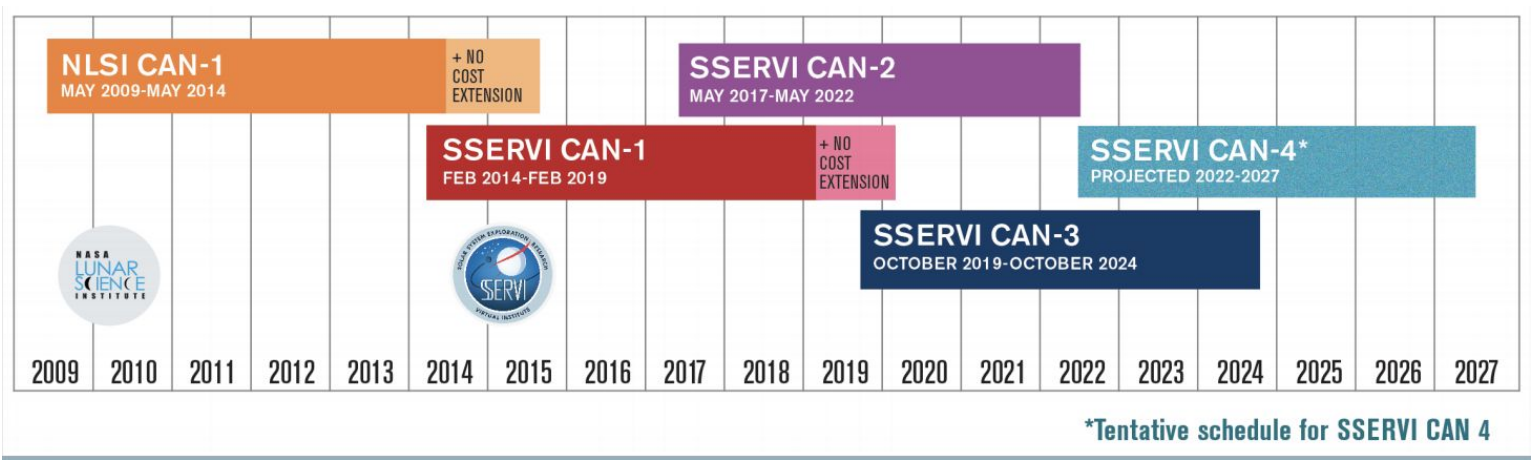

Figure 2: SSERVI Cooperative Agreement Notice (CAN) Roadmap

SSERVI provides an important interface to NASA HQ for responding to new strategic directions and providing important input to NASA. As shown several times during the history of SSERVI and NLSI, this institute has responded to changing Administration priorities first with a solely lunar emphasis, then broadening to include small bodies of potential interest for human exploration, and then refocusing much of its intent on the Moon. NASA may redirect up to $20 \%$ of team funding towards focused strategic needs, based on relevant expertise within the teams to answer NASA's questions pertinent to exploration science and provide critical help to Artemis, VIPER and other agency priorities. Frequent reporting of new discoveries are key to engaging the NASA science and exploration leadership and informing programmatic decisions.

In addition to the scientific research performed by the teams, SSERVI, in partnership with the Lunar Exploration Analysis Group (LEAG), held the "Lunar Science for Landed Missions" workshop [https://lunar-landing.arc.nasa.gov/downloads/LunarLandedScience Publication.pdf], which produced a set of high-priority targets for near-term landed missions on the Moon, in large part to provide initial guidance for NASA and commercial exploration efforts to land a wide variety of experiments on the Moon in the near term.

Additionally, through the virtual institute, the SMD Associate Administrator requested a white paper on key areas of lunar science in the new era of lunar exploration. The white paper, released in January 2018, titled "Transformative Lunar Science" [https://sservi.nasa.gov/ wp-content/uploads/2018/02/TransformativeLunarScience.pdf] provides a response to the question "What transformative lunar science issues can be addressed in the currently evolving space science era?" Led by institute and community leaders, the report was developed to explicitly address human exploration relevance, detailing key exciting lunar science efforts in the future.

The integration and implementation of virtual technologies through virtual institutes create value for NASA and the planetary science community. Virtually connecting geographically distributed teams is an essential part of a virtual institute. SSERVI has been at the leading edge 
of virtual collaboration since its inception, producing a variety of virtual presentations, large conferences, webinars, workshops without walls, seminar series, and group-to-group and person-person meetings. SSERVI Central has provided insight and guidance to numerous Agency organizations and teams to increase the awareness and effectiveness of collaboration technologies. The production of virtual events often requires an experienced technical team to work in conjunction with the scientific organizers to maximize an event's productivity. SSERVI implements and integrates high-fidelity video conferencing, real-time meeting and communication platforms, websites and dynamic web applications, online communities, social networks, shared databases, data visualization applications, and mobile devices. SSERVI also focuses on researching user experience improvements and integrating new capabilities that further enable and enhance accessibility. SSERVI has fostered commercial partnerships with its neighbors in Silicon Valley to evaluate the latest state-of-the-art in computer technology, and actively researches, reviews, and beta-tests new tools to find those best suited for virtual collaboration.

Virtual institute's international partnerships foster and strengthen common goals. As an international institute, SSERVI's 11 international partnerships [https://sservi.nasa.gov/internationals/] with foreign space agencies and institutions, on a no-exchange-of-funds basis, enables SSERVI researchers to collaborate globally to develop new science and technical approaches, benefiting the exploration science communities in both countries. SSERVI, together with its European colleagues, created the pan-European lunar consortium, and initiated the European Lunar Science workshop held annually with organizations across Europe, to help build and bridge international lunar science efforts. The SSERVI Central Office develops and maintains its international partnership program, while domestic teams nurture areas of mutual scientific interest to forge significant collaborations with international partners. Acting as a nucleating agent has been an important role for SSERVI in bringing international attention to a wide variety of NASA research interests.

NASA can leverage virtual institute research facilities to benefit targeted research efforts. Through SSERVI, a number of facilities have been developed

[https://sservi.nasa.gov/science/facilities/], some of which are unique in the world. For example, SSERVI \& NLSI funded the IMPACT team's linear electrostatic dust accelerator at the University of Colorado, Boulder, which is the only facility in the world capable of generating hypervelocity dust particles ( $>100 \mathrm{~km} / \mathrm{s}$ ). It is available to outside groups for a variety of dust impact research activities as well as calibrating dust instruments for space applications. Other unique facilities and laboratories include the Exolith lab at the University of Central Florida, which is the world's largest producer of high-fidelity planetary regolith simulants and has provided tailored simulants to over 350 unique customers, including various NASA entities. Furthermore, the SSERVI-managed testbed at NASA Ames, with 8 tons of JSC-1A lunar regolith simulant, is currently being used by the VIPER mission but is available to SSERVI teams and other NASA researchers and partners for a wide variety of research and hardware development.

Virtual institutes foster development of mission and payload concepts for later study and implementation. The virtual institute model provides a fertile ground for ideas and innovative concepts and provides a pathway for the Agency to develop these concepts in an environment 
that encourages student participation. SSERVI, in particular, does not develop missions or payloads specifically, but the scientific results have led to numerous mission and payload concepts. A few examples of how SSERVI has fostered these developments include development of the LDEX instrument concept which lead to the similar SUDA instrument for the Europa mission; the use of analog missions to conduct field work to test robotic and hand-held instrumentation for field measurements; research on voids and lava tubes for potential sustainable habitats on the Moon and Mars; work with USGS on algorithms to process remote sensing data with ground-truth to produce resource prospecting maps; development of an array of exciting astrophysics mission concepts for the Agency including, but not limited to the Farside Array for Radio Science Investigations of the Dark ages and Exoplanets (FARSIDE), Dark Ages Polarimeter Pathfinder (DAPPER), and others.

\section{Conclusion}

Virtual Institutes offer NASA a comprehensive research strategy to advance the frontiers of planetary science. SSERVI has assisted the Agency with identifying, recommending, and ranking the highest priority research activities for human exploration of the Moon. The Transformative Lunar Science whitepaper is a comprehensive review of the state-of-the-art in lunar science that was balanced with consideration for large, medium, and small research activities for both ground and space, with additional focus on sustaining global leadership in lunar exploration, establishing lunar exploration infrastructure, coordinating planning \& implementation of human/robotic partnerships, and optimizing commercial involvement. Although this document was prepared for NASA, and largely emphasizes U.S. science and exploration programs, much of the future work in lunar science will be done in collaboration with international partners. The virtual institute model expands agency potential for partnerships with foreign and U.S. agencies, and offers a comprehensive research strategy which includes the identification of new opportunities for remote cooperation. Using this model, SSERVI has identified collaborative research opportunities that are relevant to science priorities within SMD and between its science divisions (eg. identifying the location and abundance of water ice and other lunar volatiles). SSERVI research has bridged NASA SMD and the other NASA mission directorates (eg. HEOMD and research related to human exploration), NASA and other US government entities (eg. collaborative mapping efforts with USGS), NASA and private sector organizations (eg. Astrobotics, Made-In-Space, etc.) as well as NASA and its international partners. International and commercial partners have significantly contributed to Agency efforts, and clear advances in transformative planetary science have been made. Virtual institutes are high-value, low-risk funding mechanisms with broad partnership opportunities that can reduce technical risk for the Agency and help achieve technical readiness for high-priority agency programs such as Artemis. Virtual Institutes will prove helpful as NASA advances its ambitious plans for the Moon and beyond. 